\title{
Tratamientos actuales del trastorno por atracón
}

\author{
María J. González-Calderón*, Elena Ormaechea-Alegre ${ }^{\star *}$
}

Doctora en Psicología, Universidad Complutense de Madrid. Decana, Facultad de Ciencias de la Salud y la Educación, Universidad a Distancia de Madrid (Udima), Madrid, España. Correo electrónico: mariajose.gonzalez@udima.es

** Licenciada en Ciencias de la Información, Universidad Complutense de Madrid. Licenciada en Psicología, Universidad a Distancia de Madrid, España. (Udima). Correo electrónico: elenaormaechea@gmail.com

Recibido: 24 de diciembre del 2015 Aprobado: 24 de febrero del 2016

Cómo citar este artículo: GonzálezCalderón, M. J. y Ormaechea-Alegre, E. (2016). Tratamientos actuales del trastorno por atracón. Pensando Psicología, 12(19), 11-22. doi: http://dx.doi.org/10.16925/ pe.v12i19.1325

\section{Resumen}

Introducción: a pesar de que el trastorno por atracón (TA) constituye un importante problema de salud, menos de la mitad de quienes lo padecen reciben tratamiento. Objetivo: revisar la literatura reciente sobre las intervenciones llevadas a cabo en casos de TA. Método: con el fin de llevar a cabo la presente revisión narrativa, se han consultado las bases de datos Pubmed, Medline y Scielo. Solo se eligieron artículos en revistas indexadas en la Web of Science. La literatura revisada corresponde a los artículos más citados publicados en el período temporal que abarca desde el 2010 hasta el 2015. Resultados y conclusiones: los resultados muestran que las intervenciones más utilizadas son las de índole psicológica, entre las cuales se destacan la terapia cognitivo-conductual y la terapia interpersonal, recomendada esta última en pacientes con patología más severa. La terapia de conducta de pérdida de peso es menos eficaz al no reducir los atracones, por lo que no constituye el tratamiento de elección. Aunque algunos antidepresivos y antiepiléticos han mostrado cierta utilidad para reducir los atracones, el peso y patologías comórbidas, no hay evidencia de que la intervención farmacológica por sí sola sea más eficaz que la psicológica, ni de que mejore los resultados de la segunda cuando ambos se aplican conjuntamente.

Palabras clave: revisión, terapia, trastorno por atracón, tratamiento. 


\title{
Current Treatments of Binge Eating Disorder
}

\begin{abstract}
Introduction: Although binge eating disorder (BED) is a major health problem, less than half of sufferers receive treatment. Objective: To review recent literature on interventions carried out in BED cases. Method: In order to conduct this narrative review, the databases PubMed, Medline and Scielo have been consulted. Only articles in indexed journals in the Web of Science were chosen. The literature reviewed corresponds to the most cited articles published in the period between 2010-2015. Results and conclusions: The results show that the most commonly used interventions are those of psychological nature, including cognitive behavioral therapy and interpersonal therapy, the latter recommended in patients with a more severe pathology. The behavioral therapy for weight loss is less effective by not reducing binge eating; therefore, it is not the treatment of choice. Although some antidepressant and antiepileptic drugs have shown some utility in reducing binge eating, weight and comorbidities, there is no evidence that pharmacological intervention alone is more effective than psychological intervention, or that it improves the results of the latter when both are applied together.
\end{abstract}

Keywords: review, therapy, binge eating disorder, treatment.

\section{Tratamentos atuais do transtorno da compulsão alimentar periódica}

\section{Resumo}

Introdução: apesar de o transtorno da compulsão alimentar periódica (TCAP) constituir um problema de saúde importante, menos da metade dos que padecem dele recebe tratamento. Objetivo: revisar a literatura recente sobre as intervenções realizadas em casos de TCAP. Método: a fim de proceder à presente revisão narrativa, consultaram-se as bases de dados PubMed, Medline e Scielo. Somente foram escolhidos artigos em revistas indexadas na Web of Science. A literatura revisada corresponde aos artigos mais citados publicados de 2010 a 2015. Resultados e conclusões: os resultados mostram que as intervenções mais utilizadas são as de índole psicológica, entre as quais se destacam a terapia cognitivo-comportamental e a terapia interpessoal, esta última recomendada para pacientes com patologias mais graves. A terapia comportamental de perda de peso é menos eficaz ao não reduzir a compulsão alimentar, razão pela qual não constitui o tratamento de escolha. Embora alguns antidepressivos e antiepiléticos tenham mostrado certa utilidade para reduzir as compulsões, o peso e as patologias comórbidas, não há evidência de que a intervenção farmacológica por si só seja mais eficaz do que a psicológica, nem de que melhore os resultados da segunda quando ambos se aplicam conjuntamente.

Palavras-chave: revisão, terapia, transtorno da compulsão alimentar periódica, tratamento. 


\section{Introducción}

El trastorno por atracón (TA) ha recorrido un largo camino hasta ser considerado un trastorno de la alimentación con entidad nosológica propia en el recientemente publicado DSM-5. Se caracteriza por la aparición de episodios de ingesta compulsiva (atracones) de una cantidad excesiva de comida - los cuales tienen lugar en un breve espacio de tiempo-, así como por la pérdida de control sobre dicha ingesta, malestar acusado respecto a los atracones y ausencia de conductas compensatorias (APA, 2013). Su prevalencia es mayor que la de otros trastornos de la alimentación (Reas y Grilo, 2015); y se sitúa entre el 1\% y el 2\% en población general (Ágh et al., 2015; Kessler et al., 2013; Smink, van Hoeken y Hoek, 2013), si bien Hudson, Coit, Lalonde y Pope (2010) estiman que con su inclusión en el DSM-5 esta experimentará un ligero aumento, logrando alcanzar el 2,9\% en mujeres y el 3\% en hombres. Los datos existentes acerca de los efectos a largo plazo del TA, incluyendo los de mortalidad y su curso, así como estudios de seguimiento al respecto son muy escasos, a diferencia de la extensa literatura que sobre estos aspectos se posee acerca de otros trastornos de la alimentación, como la bulimia o la anorexia nerviosa (Smink, van Hoeken y Hoek, 2013). Lo que sí se conoce es que el TA es un problema común entre la población que presenta obesidad, la cual a su vez se asocia a diferentes enfermedades tales como la diabetes, la hipertensión, la hipercolesterolemia y enfermedades cardíacas que pueden conllevar serias consecuencias para la salud, afectar la calidad de vida de quienes las padecen e implicar un inadecuado funcionamiento social, tal como concluyeron Ágh et al. (2015) tras una revisión de diversas investigaciones. Estas características han llevado a la Organización Mundial de la Salud a considerar este trastorno como un importante problema de salud, si bien actualmente menos de la mitad de los sujetos que lo padecen reciben tratamiento (Kessler et al., 2013; Mond, Hay, Rodgers y Owen, 2007; Swanson, Crow, Le Grange, Swendsen y Merikangas, 2011). De hecho, el TA no empezó a tratarse como tal hasta aproximadamente la década de los 90 , fecha en la cual se incluye en el DSM-IV (APA, 1994), dentro del apéndice "Criterios y ejes propuestos para estudios posteriores". En sus inicios se emplearon las técnicas conductuales utilizadas en los individuos con obesidad, o bien estrategias cognitivo-conductuales similares a las que ya se utilizaban en el ámbito de la bulimia nerviosa (Saldaña, 2001). Sin embargo, posteriormente la investigación demostró diferencias psicopatológicas sustanciales entre ambos trastornos, por lo cual comenzaron a desarrollarse y a emplearse tratamientos específicos para cada una de las dos patologías: la bulimia y el trastorno por atracón. A día de hoy los tratamientos para el TA han evolucionado, incorporándose nuevas terapias psicológicas, tratamientos farmacológicos complementarios, así como el apoyo adicional de diversas técnicas (el mindfulness, por ejemplo), como parte de intervenciones más completas.

El objetivo del presente trabajo es revisar la literatura reciente acerca de las intervenciones que se llevan a cabo en casos de trastorno por atracón, detectar qué tratamientos son los más empleados y, sobre todo, los que resultan más eficaces según distintos meta-análisis, así como destacar los pros y contras de cada uno de ellos y cuándo resulta más conveniente emplear cada uno de los mismos. Finalmente, se comparan las distintas intervenciones a fin de que los profesionales de la salud mental conozcan qué técnicas emplear en función de las características de un determinado paciente.

\section{Método}

Para llevar a cabo la presente revisión se han consultado las bases de datos Pubmed, Medline y Scielo. La literatura revisada corresponde al período temporal que abarca desde el 2010 hasta el 2015, haciendo especial hincapié en la búsqueda de artículos publicados a partir del 2013, fecha de inclusión del trastorno por atracón en el DSM-5, con objeto de revisar si se ha incrementado la literatura e investigación al respecto e incorporar las posibles novedades. No obstante, se han referenciado algunos trabajos previos al 2010 cuyos hallazgos sobre la temática abordada resultan de especial interés. Con el fin de garantizar la calidad científica de los artículos seleccionados, se tuvo en cuenta que las revistas en las que estos se publicaron estuvieran indexadas en la Web of Science del Institute for Scientific Information (ISI), y que los artículos en cuestión se encontraran entre los más citados en su ámbito de conocimiento.

Las etiquetas de búsqueda empleadas han sido las siguientes: "binge eating disorder", "treatment", "therapy", "pharmacology", "DSM-5", "cognitive-bahavioral therapy", "interpersonal therapy" y "dialectic behavioral therapy". 


\section{El abordaje del trastorno por atracón}

Primero es importante apuntar que en la intervención del TA se deben tener en cuenta algunos aspectos que determinan la elección de la modalidad de tratamiento. Por un lado, hay que considerar el comportamiento emocional del paciente, entendido aquel como las características psicopatológicas que conducen al descontrol en la ingesta, y las consecuencias físicas de este comportamiento, las cuales en algunos casos van desde el sobrepeso hasta la obesidad (Ramacciotti et al., 2013). Por otro lado, como apunta Halmi (2013), en los trastornos de la alimentación también es necesario tener en cuenta las resistencias al tratamiento, las cuales normalmente pueden predecirse por la severidad de la patología, la cual depende a su vez de la interacción entre los factores de riesgo ambientales y una neurobiología vulnerable. Por tanto, estos dos factores - los emocionales que empujan a la ingesta compulsiva y la predisposición de un sujeto resultado de su genética y el entorno que lo rodea- son los que deben guiar la elección de una $\mathrm{u}$ otra modalidad de tratamiento.

A continuación, se exponen las principales intervenciones psicoterapéuticas utilizadas hoy en día para tratar el TA, así como los recientes avances realizados al respecto en el ámbito farmacológico.

\section{Tratamientos psicológicos del trastorno por atracón}

Las principales técnicas de intervención psicológica utilizadas en casos de TA son la terapia conductual de pérdida de peso, la terapia cognitivo-conductual y la terapia interpersonal, y en menor medida, la terapia dialéctica conductual. Existen otras técnicas, como la terapia de respuesta integrativa (Robinson, 2013), las cuales se han empleado menos y no poseen aún suficiente aval empírico, por lo que no se recogen en esta revisión.

\section{Terapia conductual de pérdida de peso}

El objetivo principal de este tratamiento es reducir la ingesta e incrementar la actividad física, a fin de conseguir una disminución del peso del paciente. Esta terapia se centra, por tanto, en la pérdida de peso más que en el atracón directamente. La intervención normalmente se realiza en sesiones de grupo y puede tener algunas ventajas respecto a otros tratamientos del
TA, tales como a) una menor necesidad de especialización, de manera que puede ser implementado por un rango de profesionales más amplio; o b) su clara focalización en el peso, aspecto que para algunos pacientes con TA puede constituir su principal objetivo o preocupación (Theim y Wilfrey, 2009). No obstante, si bien los programas de pérdida de peso ayudan a la reducción de este en los pacientes con TA que lo necesiten, muestran resultados diversos a la hora de reducir el atracón, probablemente debido a que se focalizan en la pérdida de peso y no en el atracón, como apuntan Brown y Keel (2012). Por otro lado, la eficacia de esta técnica en pacientes obesos con TA no está clara (Grilo, Masheb, Wilson, Gueorguieva y White, 2011). Grilo et al. (2011) estudiaron el empleo de la técnica de pérdida de peso seguida de la terapia cognitivo-conductual (тсC), y concluyeron que esta modalidad conjunta no es superior al empleo único de la TCC.

\section{Terapia cognitivo-conductual (TCC)}

La terapia cognitivo-conductual (тCC) es considerada uno de los tratamientos más efectivos para tratar el TA (Grilo et al., 2011; Iacovino, Gredysa, Altman y Wilfley, 2012; Ramacciotti et al., 2013; Theim y Wilfrey, 2009). Se basa en el modelo de restricción del atracón, el cual plantea que los pensamientos, creencias y preocupaciones en torno a la alimentación, el peso y la figura, promueven un patrón disfuncional de exceso y extrema restricción, de modo que el paciente alterna entre atracones y la restricción alimentaria. Por ello, el objetivo de la TCC es romper ese ciclo "atracón-dieta", sustituyendo dichos patrones alimentarios característicos del TA por otros, promoviendo patrones alimentarios más saludables y estructurados, modificando los pensamientos disfuncionales relativos a la imagen y la importancia del peso, y fomentando el uso de comportamientos saludables de control de peso. Para lograrlo se instruye a los pacientes para que no realicen una dieta antes de trabajar los objetivos de pérdida de peso, con la intención de detener el ciclo dieta-atracón. Por otro lado, la TCC incluye la modificación de aquellos pensamientos extremadamente negativos sobre el sobrepeso y la aceptación de la imagen; posteriormente, se les enseña a corregir las impresiones sobre su cuerpo, a encontrar otras formas de afrontar las situaciones y sentimientos que inducen al atracón, así como a evitar recaídas (Ramacciotti et al., 2013). 
La TCC se puede realizar de forma individual o en grupo. La modalidad individual se suele desarrollar en 14-20 sesiones una vez a la semana, si bien existen versiones más breves, y consta de tres fases (Slane y Klump, 2009): la primera se centra en ayudar al paciente a identificar los hábitos erróneos de alimentación, utilizando herramientas como los autoregistros; la segunda consiste en identificar y modificar los pensamientos disfuncionales sobre el peso y la figura; $y$ en la fase final, se trabaja la prevención de recaídas. La modalidad grupal sigue la misma estructura pero en formato de grupo. Ambas modalidades de tratamiento (individual y grupal), parecen mostrar una eficacia similar, tal como lo refleja el estudio de Ricca et al. (2010): una pérdida moderada de peso y una respuesta similar a los tres años, tanto en términos de recuperación, como de resistencia al tratamiento, recaídas y aparición de un nuevo diagnóstico. Asimismo, las dos modalidades de intervención conllevaron una reducción significativa en la frecuencia de los atracones.

En relación con la duración de la intervención, el estudio comparativo que llevaron a cabo Schlup, Meyer y Munsch (2010) acerca de la eficacia de la TCC de 16 sesiones frente a la de ocho sesiones, mostró mejores resultados en la intervención más prolongada en el tiempo. Estos autores concluyeron que, en términos generales, la TCC representa un tratamiento efectivo para el TA, si bien algunos grupos de pacientes pueden beneficiarse en mayor medida de un tratamiento más prolongado en el tiempo.

\section{Modalidad de autoayuda o guiada de la terapia cognitivo-conductual}

Con el objetivo de hacer llegar la TCC a un grupo más amplio de pacientes, se ha desarrollado una modalidad de autoayuda, la cual se caracteriza por ser un tratamiento con un menor coste y requerir menor atención por parte del especialista (Striegel-Moore et al., 2010). Dicha terapia se lleva a cabo la mayor parte de las veces a través del manual de autoayuda "Overcoming Binge Eating" de Christopher Fairburn (1995), así como mediante breves reuniones puntuales con el terapeuta. El libro de Fairburn se divide en dos partes: la primera está dirigida a la psicoeducación; en concreto, a aumentar el conocimiento que posee el paciente acerca del atracón y los trastornos asociados. La segunda parte consiste en un programa de autoayuda que describe las distintas fases de la TCC.
Al igual que la TCC estándar, la modalidad de autoayuda promueve patrones regulares de alimentación mediante autoregistros y estrategias de solución de problemas. El papel del terapeuta es fomentar la adherencia, ayudar en el desarrollo de objetivos y proveer al paciente del razonamiento que subyace a la TCC guiada (Iacovino et al., 2012).

Esta modalidad ha mostrado ser un tratamiento sólido para pacientes con TA (De Bar et al., 2013; Striegel-Moore et al., 2010). Por ello, Wilson y Zandberg (2012) argumentan la necesidad de avanzar en su difusión, al tratarse de un tratamiento breve y eficiente en costes, el cual puede ser implementado por un amplio rango de profesionales de la salud, incluyendo aquellos que no son especialistas en trastornos de la conducta alimentaria, vía contacto directo o a través de Internet. De hecho, el estudio de Wilson, Wilfley, Agras y Bryson (2010) muestra cómo este tipo de intervención puede ser implementada por profesionales de la salud con mínima experiencia terapéutica, logrando resultados similares a los obtenidos por especialistas en trastornos de la conducta alimentaria.

\section{Terapia cognitiva mejorada}

La TCC "mejorada" (Enhanced Cognitive Therapy) desarrollada por Fairburn constituye una forma más elaborada y sofisticada de este tratamiento, y sobre la cual las investigaciones iniciales sugieren que podría ser más efectiva que su versión anterior.

Esta versión mejorada utiliza una variedad de nuevas estrategias y procedimientos con el propósito de potenciar los resultados, e incluye módulos dirigidos a tratar ciertos obstáculos del cambio tales como el perfeccionismo, la baja autoestima y las dificultades interpersonales (Murphy, Straebler, Cooper y Fairburn, 2010). Cuenta con dos modalidades de tratamiento: la primera se dirige exclusivamente a tratar la psicopatología del TA; la segunda (la forma más amplia de tratamiento), no solo pone el foco de atención en la superación de los obstáculos externos de cambio, sino que también se centra en la psicopatología más compleja.

\section{Terapia interpersonal (TIP)}

La terapia interpersonal para tratar el trastorno por atracón proviene de la TIP, elaborada para intervenir en la bulimia nerviosa, y la cual a su vez tiene su origen en la utilizada para tratar la depresión. 
A diferencia de la TCC, en lugar de focalizar la atención en los patrones de pensamiento y comportamiento, la TIP se centra en los problemas del ámbito interpersonal del paciente (Theim y Wilfrey, 2009). Este enfoque plantea que las dificultades interpersonales son comunes en los pacientes con trastornos de alimentación, y además parecen contribuir al mantenimiento de dichos trastornos a través de diferentes mecanismos. Los pacientes con TA frecuentemente se aíslan de su entorno social y, como consecuencia, su patología persiste; asimismo, algunos rasgos del trastorno por atracón podrían estar directamente mantenidos por dificultades interpersonales, las cuales frecuentemente sirven para empeorar la autoestima (Murphy, Straebler, Basden, Cooper y Fairburn, 2012).

Por ello, el objetivo de la TIP consiste en reducir la patología del atracón basándose en el desarrollo de habilidades interpersonales saludables, las cuales puedan reemplazar los comportamientos desadaptativos y promover una autoimagen saludable.

La TIP suele tener una duración de entre 16 y 20 sesiones (4-5 meses), de 50 minutos cada una aproximadamente. Comprende tres fases, cada una con sus propios objetivos y estrategias (Murphy et al., 2012). En la primera, el objetivo es comprometer al paciente con el tratamiento y describir sus fundamentos, es decir, que los estados emocionales negativos consecuencia de los problemas interpersonales son los que activan los atracones; a continuación, se identifican las dificultades interpersonales implicadas en el trastorno. En la segunda fase se busca que el paciente comprenda la naturaleza de los problemas identificados y trabaje sobre ellos. La última fase tiene como objetivo evaluar los progresos, asegurarse del mantenimiento de estos y prevenir las recaídas.

Parece existir evidencia empírica sobre la eficacia de la TIP en la reducción del TA, y en muchos casos, con resultados similares a los obtenidos por la TCC (Brown y Keel, 2012).

\section{Terapia dialéctica conductual (TDC)}

Esta terapia plantea que las dificultades en la regulación del afecto son las que pueden conducir a comportamientos patológicos o desadaptativos, como el atracón. Se basa en el modelo de regulación del afecto del atracón, según el cual este ocurre como respuesta a experiencias emocionales adversas cuando los mecanismos adaptativos para manejarlas no están disponibles. El atracón actúa así como un alivio temporal del afecto negativo, reforzando el comportamiento, en este caso el atracón. Wallace, Masson, Safer y von Ranson (2014) confirmaron el papel de la regulación de las emociones en la consecución de la abstinencia de los atracones, y concluyeron que la modificación de dichas emociones al final del tratamiento predice la abstinencia del atracón en los pacientes que reciben un tratamiento con TDC.

En un estudio inicial de Telch, Agras y Linehan (2001) en la Universidad de Standford, la TDC adaptada para el TA mostró resultados prometedores en una muestra de mujeres, al alcanzarse una significativa mejoría en los atracones, así como en la patología alimentaria, en comparación con el grupo de control. Otras investigaciones posteriores corroboran que la TDC puede ser una terapia efectiva para tratar el TA (Klein, Skinner y Hawley, 2013; Masson, von Ranson, Wallace y Safer, 2013; Robinson y Safer, 2011; Roosen, Safer, Adler, Cebolla y van Strien, 2012).

Los trabajos incluidos en la revisión de Iacovino et al. (2012) sobre los tratamientos psicológicos eficaces para el TA también muestran significativas tasas de abstinencia de atracones asociadas al tratamiento con TDC. Sin embargo, estos autores sugieren que aunque los resultados son prometedores, es necesaria una mayor investigación al respecto a fin de examinar la eficacia a largo plazo de la TDC comparada con otros tratamientos.

Uno de los cuatro componentes de la TDC es el mindfulness, técnica empleada para mejorar el reconocimiento o la consciencia de los atracones, incrementar el autocontrol y mantener una actitud de autoaceptación. Existen adaptaciones de esta técnica al tratamiento concreto del TA, entre las que se destaca el "Entrenamiento para adquirir conciencia de la ingesta para tratar el TA basado en el mindfulness" (Kristeller y Wolever, 2011), el cual comprende un entrenamiento en meditación y una guía de prácticas de mindfulness diseñada para centrarse en las cuestiones básicas del TA (controlar las respuestas a los estados emocionales variables, ser consciente de las elecciones de alimentos, concienciarse de las señales de hambre y saciedad presentes, y cultivar la autoaceptación).

El mindfulness ha mostrado ser beneficioso para tratar el TA, no solo como parte de la TDC, sino también cuando se añade a otras modalidades de tratamiento, como la TCC (Courbasson, Nishikawa y Shapira, 2011; Woolhouse, Knowles y Crafti, 2012). El reciente metaanálisis de Godfrey, Gallo y Afari (2014) recoge de forma sistemática el impacto positivo de las 
intervenciones basadas en el mindfulness para tratar el TA.

\section{Tratamientos farmacológicos del trastorno por atracón}

Además de los tratamientos de carácter psicológico, también se ha propuesto el empleo de determinados fármacos para combatir los atracones y, por ende, el trastorno por atracón. Según McElroy, Guerdjikova, Mori y O'Melia (2012), los principales argumentos a favor del uso de la farmacoterapia para tratar el TA serían los siguientes: a) los pacientes con TA no siempre responden adecuadamente a las intervenciones psicológicas, por lo que quizá algunos pacientes crónicos o más graves necesiten medicación para mejorar los resultados de dichos tratamientos; b) el TA se asocia con algunas alteraciones neurobiológicas, como, por ejemplo, disfunciones en algunos transmisores tales como la dopamina, o en el sistema serotoninérgico; y c) algunos medicamentos tienen efectos sobre el apetito y el peso, de manera que pueden contribuir a mejorar los efectos terapéuticos de otros tratamientos no farmacológicos.

Los tres tipos de medicación más habituales en el tratamiento del TA son los antidepresivos (especialmente los Inhibidores Selectivos de la Recaptación de Serotonina o ISRS, como la fluoxetina o la fluoxamina), los antiepilépticos (especialmente el topiramato), y los fármacos contra la obesidad, siendo los primeros los que parecen más prometedores (Marazziti, Corsi, Baroni, Consoli y Catena-Dell'Osso, 2012).

McElroy et al. (2012) realizaron una extensa revisión sobre los diferentes tratamientos farmacológicos para tratar el TA y concluyeron que la mayoría de estos cuentan con limitaciones importantes, tales como el empleo de muestras pequeñas, una alta respuesta al placebo, altas tasas de abandono de los tratamientos y la dificultad que implica trasladar los resultados del laboratorio al mundo real. A pesar de las limitaciones enumeradas, estos autores consideran que existe suficiente evidencia acerca de una eficacia moderada de los antidepresivos en el tratamiento del TA a corto plazo, si bien estos fármacos no modifican el peso del paciente y se desconocen sus resultados a largo plazo.

Ramacciotti et al. (2013) analizaron precisamente los resultados de diversos estudios que emplearon antidepresivos y extrajeron las siguientes conclusiones: a) respecto a los ISRS, la administración de fluoxetina y fluvoxamina parece asociarse con una reducción de la frecuencia del atracón, además de una disminución de la sintomatología depresiva que puede estar presente en algunos casos de TA; b) los antidepresivos tricíclicos, como la imipramina, también resultan eficaces a la hora de reducir los episodios de atracón, así como el peso y un posible estado depresivo; y c) en relación con los Inhibidores de la Recaptación de Serotonina y Noradrenalina (IRSN), la duloxetina parece mejorar significativamente no solo la sintomatología depresiva, sino también la específica del TA. Trabajos posteriores han constatado que algunos fármacos antiepilépticos como el topiramato o la topiramida resultan efectivos en la reducción del atracón, así como en la pérdida de peso, además de disminuir la patología obsesivo compulsiva o los rasgos de impulsividad asociados (Kimberly, Brownley, Peat, La Via y Bulik, 2015; Ramacciotti et al., 2013; Reas y Grilo, 2014).

Por último, cabe añadir que la Federal Drugs Administration (FDA) ha aprobado en enero del 2015 el uso del primer fármaco especialmente dirigido al tratamiento del TA, la lisdexamfetamina, cuya forma comercial es Vyvanse (Cassels, 2015). Según la FDA, la eficacia de este fármaco ha sido probada en dos estudios, cuyos participantes mostraron una disminución en el número de atracones, así como una reducción de los comportamientos obsesivo-compulsivos del atracón (Citrome, 2015).

\section{Eficacia comparativa de los distintos tratamientos para el TA}

Wilson et al. (2010) realizaron el primer estudio controlado para comparar distintos tratamientos psicológicos utilizados con el fin de tratar el trastorno por atracón. Los pacientes recibieron aleatoriamente veinte sesiones de terapia de pérdida de peso, impartida por especialistas en psicología clínica, o veinte sesiones de TIP impartida por médicos terapeutas, o bien diez sesiones de TCC en la modalidad de autoayuda (guiada), llevada a cabo por estudiantes sin experiencia en TCC ni en el tratamiento del TA, quienes habían sido entrenados mediante un taller de tres horas utilizando el manual de Fairburn. A los dos años de seguimiento, el TA había disminuido significativamente en los grupos que emplearon tanto TIP como TCC guiada, pero no en el grupo de terapia de pérdida de peso, siendo los pacientes con baja autoestima y síntomas más severos los que respondieron particularmente mal a dicha terapia. Estos autores concluyeron que la TCC debería ser el tratamiento de 
elección para la mayoría de los pacientes con TA, seguida de la TIP; de igual forma, esta última terapia debería ser el tratamiento preferente en aquellos pacientes que presentaran síntomas más severos.

Posteriormente, Hilbert et al. (2012) también demostraron la efectividad de la TCC y la TIP en un estudio de seguimiento a largo plazo de cuatro años, en el cual examinaron la eficacia de dichos tratamientos administrados a dos grupos de pacientes con TA. Todos los participantes mostraron una sustancial recuperación, significativa mejoría clínica y reducción de su psicopatología, si bien su IMC se mantuvo estable. Los resultados del estudio también mostraron la eficacia a largo plazo de ambos tratamientos, ya que el $64,4 \%$ de los pacientes se recuperaron por completo. De acuerdo con la revisión de Brown y Keel (2012), los mejores tratamientos actuales hasta la fecha para el TA también incluyen la TCC y la TIP. Las tasas de remisión para el TA aplicando uno de estos tratamientos son más altas que para la anorexia o la bulimia: aproximadamente en el $82 \%$ de los individuos tratados con TCC y el $80 \%$ de los tratados con TIP se consigue la remisión del trastorno.

Iacovino et al. (2012) realizaron una revisión más amplia con el objetivo de comparar la eficacia de un mayor número de terapias, la TCC, la TCC guiada, la TIP, la TCD y la terapia de conducta de pérdida de peso, concluyendo que la TCC era la terapia más estudiada y con mayor evidencia empírica para el tratamiento psicológico del TA. Asimismo, los resultados mostraron que la TCC, tanto en grupo como individual, y la TCC guiada, junto con la TIP, presentan los resultados más sólidos a corto y largo plazo, al asociarse con tasas de abstinencia de atracón más altas. Estos autores, al igual que hicieron previamente Wilson el al. (2010), también concluyeron que la TIP mostraba una mayor eficacia en pacientes con patología más severa y menor autoestima. En la misma línea, la revisión posterior de Ramacciotti et al. (2013) refuerza la evidencia empírica sobre la eficacia de la TCC en el tratamiento del TA y sugiere que los pacientes con alta afectividad negativa y una clínica similar a la bulimia nerviosa podrían beneficiarse más de la TIP. Asimismo, estos autores plantean que uno de los retos actuales en el tratamiento del TA es el control del peso, ya que ni la TCC ni la TIP han mostrado ser clínicamente relevantes en la pérdida o mantenimiento del peso, ni en la prevención de recaídas en este aspecto.

En distintos estudios la modalidad de autoayuda de la TCC no solo ha obtenido resultados similares a la TIP y superiores a otros tratamientos - como la terapia de conducta de pérdida de peso-, sino que ha resultado ser más efectiva en costes que el tratamiento habitual, tal como demostraron Lynch et al. (2010) empleando el método CEA (Cost-Effective Analysis).

Los estudios que se han llevado a cabo en población adolescente que padece TA reflejan resultados dispares. Por un lado, el estudio de Tanofsky-Kraff et al. (2009) sugería que la TIP era una terapia factible para tratar el TA en esta etapa evolutiva; por otro lado, el estudio de DeBar et al. (2013) sugería que la TCC poseía una potencial eficacia para este tipo de pacientes, como se ha encontrado repetidamente en población adulta; sin embargo, el trabajo de Bailey, Parker, Colautti, Hart, Liu y Hetrick (2014) concluía que existen pocos ensayos sobre la eficacia del TCC en adolescentes y, aunque se detectaban efectos positivos, los resultados no eran concluyentes. La revisión más actual y extensa acerca de los tratamientos eficaces para tratar el TA en población adolescente es la de Lock (2015), que sitúa en el nivel de tratamientos "probablemente eficaces" a la TCC autoguiada y en el nivel de tratamientos "experimentales" a la TDC.

Como puede extraerse de los estudios enumerados, aunque la terapia conductual de pérdida de peso ha mostrado ser superior a la TCC y la TIP en la reducción del IMC y del peso, así como en el mantenimiento de la dieta (Brown y Keel, 2012; Wilson et al., 2010), son múltiples las investigaciones que consideran que no puede ser el tratamiento de elección para el TA, pues no consigue reducir la frecuencia de los atracones, síntoma central del TA. Asimismo, conviene destacar que aquellos pacientes caracterizados por una patología más severa responden mejor a los tratamientos específicos, como la TCC y la TIP, en comparación con los pacientes tratados con la terapia de pérdida de peso (Sysko, Hildebrandt, Wilson, Wilfley y Agras, 2010).

En relación con el empleo de fármacos para tratar el TA, existen discrepancias respecto a su eficacia en solitario, así como acerca del incremento de la mejoría del trastorno cuando se añade el tratamiento farmacológico al psicológico. En esta línea, Grilo, Crosby, Wilson y Masheb (2012) examinaron los efectos a largo plazo de tres tratamientos diferentes: solo fluoxetina, fluoxetina junto con una intervención terapéutica (TCC), у TCC con un placebo. Los resultados de este último grupo (TCC con un placebo) fueron superiores a los de los grupos que recibieron fluoxetina (sola o junto con la TCC). Asimismo, Grilo, Masheb y Crosby (2012) también detectaron que los 
pacientes que recibían un tratamiento con TCC presentaban mayores tasas de remisión del trastorno, y una mayor reducción en los niveles de depresión que los pacientes que solo recibían tratamiento farmacológico. Del mismo modo, Brown y Keel (2012) también concluyeron que no existen evidencias respecto a una mayor eficacia del tratamiento cuando se añade la intervención farmacológica a la TCC. Estos resultados coinciden con la revisión de Hofmann, Asnaani, Vonk, Sawyer y Fang (2012), quienes sugieren que la combinación de la psicoterapia y la medicación no mejora los resultados respecto del atracón, aunque sí puede mejorar los relativos a la pérdida de peso.

Por otro lado, Reas y Grilo (2014) analizaron los estudios controlados de diferentes tratamientos farmacológicos y concluyeron que existe una carencia de datos referentes a la eficacia a largo plazo de la medicación, con la única excepción del topiramato - ya comentada-, el cual sí parece reducir tanto el atracón, como el peso. No obstante, estos autores afirman que tanto el tratamiento psicológico, como la combinación de este junto con la medicación, producen resultados superiores al empleo de fármacos en solitario y, si bien combinar la medicación con las intervenciones psicológicas no mejora significativamente los resultados, la utilización de ciertos medicamentos puede mejorar los logros de la terapia de pérdida de peso, así como los de la pérdida de peso alcanzada con la terapia cognitivo conductual.

\section{Conclusiones}

Las intervenciones que se han utilizado en mayor medida para tratar el trastorno por atracón son las terapias de índole psicológica. En este aspecto, las que muestran una mayor evidencia empírica respecto a su eficacia son la TCC, incluida su modalidad de autoayuda, y la TIP, como concluyen numerosas revisiones llevadas a cabo en los últimos años (Hilbert et al., 2012; Iacovino et al., 2012; Wilson et al, 2010). La TCC, especialmente su modalidad de autoayuda, presenta la ventaja de ser un tratamiento eficaz con un coste menor (Lynch et al., 2010), y una implementación más fácil, mientras que la TIP parece ser más recomendable en aquellos pacientes con una patología más severa (Iacovino et al., 2012; Wilson et al., 2010), o en los que presentan una alta afectividad negativa y una clínica similar a la bulimia nerviosa (Ramacciotti et al., 2013).

Sin embargo, estas terapias presentan algunas limitaciones respecto a su efecto en la reducción y el mantenimiento del peso (Ramacciotti et al., 2013). Por el contrario, la terapia de conducta de pérdida de peso, aunque sí muestra resultados satisfactorios en el objetivo de disminuir el peso, y por ende el IMC, presenta una menor eficacia como terapia específica dirigida al TA, ya que no reduce los atracones, por lo que no debe proponerse como tratamiento de elección, especialmente en aquellos sujetos que presentan una patología más severa (Sysko et al., 2010).

Por otro lado, aunque la TDC muestra algunos resultados prometedores (Klein et al., 2013; Masson et al., 2013; Robinson y Safer, 2011; Roosen et al., 2012; Telch et al., 2001), su superioridad frente a la TCC y la TIP no se ha demostrado hasta la fecha, por lo que es necesaria una mayor investigación al respecto, como sugirieron Iacovino et al. (2012).

En la adolescencia, etapa en la que resulta imprescindible emplear los tratamientos más eficaces que impidan que el trastorno se cronifique y, por tanto, su remisión sea menos probable, los resultados obtenidos en distintos estudios son similares a los obtenidos en adultos (DeBar et al., 2013; Lock, 2015; TanofskyKraff et al., 2009). Sin embargo, existe poca literatura al respecto, tal como apuntan Bailey et al. (2014), considerándose la TDC un tratamiento únicamente experimental (Lock, 2015), poco empleado y estudiado aún en esta etapa evolutiva tan crucial.

En cuanto a los tratamientos farmacológicos para tratar el TA, si bien los estudios al respecto presentan importantes limitaciones (McElroy et al., 2012), - entre ellas la ausencia de datos sobre su eficacia a largo plazo (Reas y Grilo, 2014)_-, parece que la utilización de algunos antidepresivos y antiepilépticos ha mostrado cierta utilidad a la hora de reducir los atracones y el peso, además de disminuir determinadas patologías comórbidas del paciente (Kimberly et al., 2015; Marazziti et al., 2012; McElroy et al., 2012; Ramacciotti et al., 2013; Reas y Grilo, 2014). Sin embargo, no hay evidencia empírica de que la intervención farmacológica por sí sola sea más eficaz que el tratamiento psicológico, ni de que esta mejore los resultados de la terapia psicológica cuando ambos se aplican conjuntamente (Brown y Keel, 2012; Grilo et al., 2012; Kass, Kolko y Wilfley, 2013). En ciertas revisiones se ha concluido incluso que las intervenciones psicológicas permiten obtener mejores resultados que la medicación o la combinación de ambos tratamientos (Grilo et al., 2012), o que, en todo caso, cualquier tratamiento conjunto es preferible al empleo de fármacos en solitario (Reas y Grilo, 2014), ya que, aunque la combinación de psicoterapia y medicación no 
mejora significativamente los logros obtenidos con la primera, sí puede influir positivamente en la pérdida de peso en comparación con la intervención psicológica en solitario.

Teniendo en cuenta los datos proporcionados se podría concluir que si bien la farmacología puede tener un importante papel en el manejo del TA, en aquellos pacientes con otros trastornos psiquiátricos, o los que presentan una enfermedad crónica o intratable - especialmente en los que responden inadecuadamente a la psicoterapia-, los fármacos empleados en la actualidad para tratar el TA están lejos de ser los más adecuados si se tienen en cuenta las distintas evidencias, tal y como ya apuntaban McElroy et al. (2012).

\section{Referencias}

Ágh, T., Kovács, G., Pawaskar, M., Supina, D., Inotai, A. y Vokó, Z. (2015). Epidemiology, health-related quality of life and economic burden of binge eating disorder: a systematic literature review. Eating and Weight Disorders, 20(1), 1-12. doi: 10.1007/s40519-014-0173-9

American Psychiatric Association. [APA]. (1994). Diagnostic and statistical manual of mental disorders. DSM-IV ( $4^{\mathrm{a}}$ ed). Washington, DC: American Psychiatric Publishing.

American Psychiatric Association. [APA]. (2013). Feeding and eating disorders. Arlington, va: American Psychiatric Publishing.

Bailey, A., Parker, A., Colautti, L., Hart, L., Liu, P. y Hetrick, S. (2014). Mapping the evidence for the prevention and treatment of eating disorders in young people. Journal of Eating Disorders, 2(1), 5.

Brown, T. A. y Keel, P. K. (2012). Current and emerging directions in the treatment of eating disorders. Substance Abuse: Research and Treatment, 6, 33-61. doi: 10.4137/ SART.S7864

Cassels, C. (2015). FDA okays Vyvanse for binge eating disorder. Medscape. Recuperado de: http://www.medscape. com/viewarticle/839039

Citrome, L. (2015). Lisdexamfetamine for binge eating disorder in adults: a systematic review of the efficacy and safety profile for this newly approved indication-what is the number needed to treat, number needed to harm and likelihood to be helped or harmed? International Journal of Clinical Practice, 69(4), 410-421. doi: 10.1111/ijcp.12639

Courbasson, C., Nishikawa, Y. y Shapira, L. (2011). Mindfulness-action based cognitive behavioral therapy for concurrent binge eating disorder and substance use disorders. Eating Disorders, 19(1), 17-33. doi: 10.1080/10640266.2011.533603

DeBar, L. L., Wilson, G. T., Yarborough, B. J., Burns, B., Oyler, B., Hildebrandt, T., et al. (2013). Cognitive behavioral treatment for recurrent binge eating in adolescent girls: A pilot trial. Cognitive and Behavioral Practice, 20(2), 147-161. doi: 10.1016/j.cbpra.2012.04.001

Fairburn, C. G. (1995). Overcoming Binge Eating. New York: Guilford Press.

Godfrey, K., Gallo, L. y Afari, N. (2014). Mindfulness-based interventions for binge eating: a systematic review and meta-analysis. Journal of Behavioral Medicine, 38(2), 348-362. doi: 10.1007/s10865-014-9610-5

Grilo, C. M., Crosby, R. D., Wilson, G. T. y Masheb, R. M. (2012). 12-month follow-up of fluoxetine and cognitive behavioral therapy for binge eating disorder. Journal of Consulting and Clinical Psychology, 80(6), 1108-1113. doi: 10.1037/a0030061

Grilo, C. M., Masheb, R. M. y Crosby, R. D. (2012). Predictors and moderators of response to cognitive behavioral therapy and medication for the treatment of binge eating disorder. Journal of Consulting and Clinical Psychology, 80 (5), 897-906.

Grilo, C. M., Masheb, R. M., Wilson, G. T., Gueorguieva, R. y White, M. A. (2011). Cognitive-behavioral therapy, behavioral weight loss, and sequential treatment for obese patients with binge-eating disorder: a ramdomized controlled trial. Journal of Consulting and Clinical Psychology, 79(5), 675-685. doi: 10.1037/a0025049

Halmi, K. (2013). Perplexities of treatment resistence in eating disorders. BMC Psychiatry, 13(1), 292. doi:10.1186/1471-244X-13-292

Hilbert, A., Bishop, M. E., Stein, R. J., Tanofsky-Kraff, M., Swenson, A. K., Welch, R. et al. (2012). Long-term efficacy of psychological treatments for binge eating disorder. The British Journal of Psychiatry: The Journal of Mental Science, 200(3), 232-237. doi: 10.1192/bjp. bp. 110.089664

Hofmann, S., Asnaani, A., Vonk, I., Sawyer, A. y Fang, A. (2012). The efficacy of cognitive behavioral therapy: A review of meta-analyses. Cognitive Therapy and Research, 36(5), 427-440. doi: 10.1007/s10608-012-9476-1

Hudson, J., Coit, C., Lalonde, J. y Pope, H. (2010). By how much will the proposed new DSM- 5 criteria increase the prevalence of binge eating disorder? The International Journal of Eating Disorders, 45(1), 139-141. doi: 10.1002/eat.20890 
Iacovino, J. M., Gredysa, D. M., Altman, M. y Wilfley, D. E. (2012). Psychological treatments for binge eating disorder. Current Psychiatry Reports, 14(4), 432-446. doi: 10.1007/s11920-012-0277-8

Kass, A., Kolko, R. y Wilfley, D. (2013). Psychological treatments for eating disorders. Current Opinion in Psychiatry, 26(6), 549-555. doi: 10.1097/YCO. 0b013e328365a30e

Kessler, R., Berglund, P., Chiu, W., Deitz, A., Hudson, J., Shahly, V., et al. (2013). The prevalence and correlates of binge eating disorder in the World Health Organization World Mental Health Surveys. Biological Psychiatry, 73(9), 904-914. doi:10.1016/j.biopsych.2012.11.020

Kimberly, A., Brownley, K., Peat, C., La Via, M. y Bulik, C. (2015). Pharmacological approaches to the management of binge eating disorder. Drugs, 75(1), 9-32. doi: 0.1007/s40265-014-0327-0

Klein, A. S., Skinner, J. B. y Hawley, K. M. (2013). Targeting binge eating through components of dialectical behavior therapy: Preliminary outcomes for individually supported diary card self-monitoring versus group-based DBT. Psychotherapy, 50(4), 543-552. doi: $10.1037 / \mathrm{a} 0033130$

Kristeller, J. L. y Wolever, R. Q. (2011) Mindfulness-based eating awareness training for treating binge eating disorder: The conceptual foundation. Eating Disorders, 19(1), 49-61. doi: 10.1080/10640266.2011.533605

Lock, J. (2015). An update on evidence-based psychosocial treatments for eating disorders in children and adolescents. Journal of Clinical Child and Adolescent Psychology, 44(5), 707-721. doi: 10.1080/15374416.2014.971458

Lynch, F., Striegel-Moore, R., Dickerson, J., Perrin, N., DeBar, L., Wilson, G., et al. (2010). Cost-effectiveness of guided self-help treatment for recurrent binge eating. Journal of Consulting and Clinical Psychology, 78(3), 322-333. doi: 10.1037/a0018982

McElroy, S. L., Guerdjikova, A. J., Mori, N. y O’Melia, A. M. (2012). Pharmacological management of binge eating disorder: current and emerging treatment options. Therapeutics and Clinical Risk Management, 8, 219. 241. doi: 10.2147/TCRM.S25574

Marazziti, D., Corsi, M., Baroni, S., Consoli, G. y Catena-Dell'Osso, M. (2012). Latest advancements in the pharmacological treatment of binge eating disorder. European Review for Medical and Pharmacological Sciences, 16(15), 2102-2107.

Masson, P. C., von Ranson, K. M., Wallace, L. M. y Safer, D. L. (2013). A randomized wait-list controlled pilot study of dialectical behaviour therapy guided self-help for binge eating disorder. Behaviour Research and Therapy, 51(11), 723-728. doi: 10.1016/j.brat.2013.08.001

Mond, J. M., Hay, P. J., Rodgers, B. y Owen, C. (2007). Health service utilization for eating disorders: findings from a community-based study. The International Journal of Eating Disorders, 40(5), 399-408. doi: 10.1002/ eat. 20382

Murphy, R., Straebler, S., Basden, S., Cooper, Z. y Fairburn, C. G. (2012). Interpersonal psychotherapy for eating disorders. Clinical Psychology and Psychotherapy, 19(2), 150-158. doi: 10.1002/cpp.1780

Murphy, R., Straebler, S., Cooper, Z. y Fairburn, C. G. (2010). Cognitive behavioral therapy for eating disorders. The Psychiatric Clinics of North America, 33(3), 611-627. doi: 10.1016/j.psc.2010.04.004

Ramacciotti, C., Coli, E., Marazziti, D., Segura-García, C., Brambilla, F., Piccinni, A., et al. (2013). Therapeutic options for binge eating disorder. Eating and Weight Disorders, 18(1), 3-9. doi:10.1007/s40519-013-0003-5

Reas, D. L. y Grilo, C. M. (2014). Current and emerging drug treatments for binge eating disorder. Expert Opinion on Emerging Drugs, 19(1), 99-142. doi: $10.1517 / 14728214.2014 .879291$

Reas, D. L. y Grilo, C. M. (2015). Pharmacological treatment of binge eating disorder: update review and synthesis. Expert Opinion on Pharmacotherapy, 16(10), 1463 1478. doi: 10.1517/14656566.2015.1053465

Ricca, V., Castellini, G., Mannucci, E., Lo Sauro, C., Ravaldi, C., Rotella, C., et al. (2010). Comparison of individual and group cognitive behavioral therapy for binge eating disorder. A randomized, three-year follow-up study. Appetite, 55(3), 656-665. doi: 10.1016/j. appet.2010.09.019

Robinson, A. (2013). Integrative Response Therapy for Binge Eating Disorder. Cognitive and Behavioral Practice, 20(1), 93-105. doi:10.1016/j.cbpra.2012.02.004

Robinson, A. y Safer, D. (2011). Moderators of dialectical behavior therapy for binge eating disorder: Results from a randomized controlled trial. The International Journal of Eating Disorders, 45(4), 597-602. doi: 10.1002/ eat.20932

Roosen, M. A., Safer, D., Adler, S., Cebolla, A. y van Strien, T. (2012). Group dialectical behavior therapy adapted for obese emotional eaters; a pilot study. Nutrición Hospitalaria, 27(4), 1141-1147. doi: 10.3305/ nh.2012.27.4.5843

Saldaña, C. (2001). Tratamientos psicológicos eficaces para trastornos del comportamiento alimentario. Psicothema, 13(3), 381-392. 
Schlup, B., Meyer, A. y Munsch, S. (2010). A non-randomized direct comparison of cognitive-behavioral shortand long-term treatment for binge eating disorder. Obesity Facts, 3(4), 261-266. doi: 10.1159/000319538

Slane, J. D. y Klump, K. L. (2009). Cognitive-behavioral therapy for binge eating disorder. En N. Chambers (ed.), Binge eating: psychological factors, symptoms and treatment (pp. 209-220). Nueva York: Nova Science Publishers.

Smink, F., van Hoeken, D. y Hoek, H. (2013). Epidemiology, course, and outcome of eating disorders. Current Opinion in Psychiatry, 26(6), 543-548. doi: 10.197/YCO. 0bo13e328365a24f

Striegel-Moore, R., Wilson, G., DeBar, L., Perrin, N., Lynch, F., Rosselli, F., et al. (2010). Cognitive behavioral guided self-help for the treatment of recurrent binge eating. Journal of Consulting and Clinical Psychology, 78(3), 312-321. doi: 10.1037/a0018915

Swanson, S. A., Crow, S. J., Le Grange, D., Swendsen, J. y Merikangas, K. R. (2011). Prevalence and correlates of eating disorders in adolescents. Results from the national comorbidity survey replication adolescent supplement. Archives of General Psychiatry, 68(7), 714-723. doi: 10.1001/archgenpsychiatry.2011.22

Sysko, R., Hildebrandt, T., Wilson, G., Wilfley, D. y Agras, W. (2010). Heterogeneity moderates treatment response among patients with binge eating disorder. Journal of Consulting and Clinical Psychology, 78(5), 681-690. doi: 10.1037/a0019735

Tanofsky-Kraff, M., Wilfley, D., Young, J., Mufson, L., Yanovski, S., Glasofer, D., et al. (2009). A pilot study of interpersonal psychotherapy for preventing excess weight gain in adolescent girls at-risk for obesity. The International Journal of Eating Disorders, 43(8), 701 706. doi: 10.1002/eat.20773

Telch, C., Agras, W. y Linehan, M. (2001). Dialectical behavior therapy for binge eating disorder. Journal of Consulting and Clinical Psychology, 69(6), 1061-1065. doi:10.1037/0022-DD6X.69.6.1061

Theim, K. R. y Wilfrey, D. E. (2009). Psychological treatment of binge eating disorder in adults. En N. Chambers (ed.), Binge eating: psychological factors, symptoms and treatment (pp. 185-202). Nueva York: Nova Science Publishers.

Wallace, L., Masson, P., Safer, D. y Von Ranson, K. (2014). Change in emotion regulation during the course of treatment predicts binge abstinence in guided selfhelp dialectical behavior therapy for binge eating disorder. Journal of Eating Disorders, 2(1), 35. doi: 10.1186/s40337-014-0035-X

Wilson, G., Wilfley, D., Agras, W. y Bryson, S. (2010). Psychological treatments of binge eating disorder. Archives of General Psychiatry, 67(1), 94-101. doi: 10.1001/ archgenpsychiatry.2009.170

Wilson, G. T. y Zandberg, L. J. (2012). Cognitive-behavioral guided self-help for eating disorders: effectiveness and scalability. Clinical Psychology Review, 32(4), 343-357. doi: 10.1016/j.cpr.2012.03.001

Woolhouse, H., Knowles, A. y Crafti, N. (2012). Adding mindfulness to Свт programs for binge eating: A mixed-methods evaluation. Eating Disorders, 20(4), 321-339. doi: 10.1080/10640266.2012.691791 